\title{
Hepatocellular carcinoma and strongyloides infection with portal vein thrombosis
}

\begin{abstract}
More than $80 \%$ of the primary liver cancers are hepatocellular carcinomas (HCC) and these tumors occurs in livers that are cirrhotic due to, for example, chronic hepatitis B (HBV) or $\mathrm{C}(\mathrm{HCV})$ viral infections.

We report a case of a 64-year-old Egyptian male who was admitted to our hospital with fever of unknown origin and elevated liverenzyms. He is known with HCV and multifocal HCC. He was treated for the HCC with a partial left liver resection and radiofrequent ablation (RFA), recently. Eventually there appeared to be an underlying strongyloides infection which causes portal vein thrombosis with general malaise, elevated liverenzyms and ascites. After treatment of the strongyloides infection his laboratory results normalized and his symptoms disappeared.
\end{abstract}

Volume 4 Issue | - 2018

\section{Marije Vlug}

Maag-Darm-en Leverarts, Netherlands

Correspondence: Marije Vlug, Maag-Darm-en Leverarts, Westfriesgasthuis, Maelsonstraat 3, I624 NP, Hoorn,

Netherlands, Tel 0229208212

Emailm.s.vlug@westfriesgasthuis.nl

Received: October 28, 2016 | Published: January 29, 2018

Keywords: strongyloides infection, portal vein thrombosis, HCC

Abbreviations: HCC, hepatocellular carcinoma; HBV, hepatitis $\mathrm{b}$ virus infection; HCV, hepatitis c virus infection; RFA, radiofrequent ablation; AF, alkalic fosfatase; GGT, gamma-glutamyltransferase; CT, abdominal computerized tomography; MRI, magnetic resonance imaging

\section{Case report}

A few years ago 64-year-old Egyptian male was admitted to our gastro-enterology department because of fever. He is known with HCV and hepatocellular carcinoma for which he was operated on in 2008. This operation enhanced a partial left liver resection, but revealed to be irradical. He received RFA additionally. Now he presented himself with ongoing fever, antibiotics had no effect.

Laboratory showed elevated liverenzyms, especially cholestatic enzymes (AF en GGT) and elevated alpha-fetoprotein. Ultrasound en X-rays showed no abnormalities. During his stay in the hospital he deteriorates and developed ascites. And additional CT scan revealed a portal vein thrombosis (Figure 2). We could not find any other explanation for his complaints than recidive tumor with fever due to tumor necrosis. He was discharged from the hospital after 2 weeks and would be followed up on the outpatient clinic. He should receive palliative chemotherapy. Than in the outpatient clinic the serology of strongyloides and schistosomiasis became positive. The schistosomiasis infection is chronic. He was treated for the strongyloides infection with Ivermectine (one dose) and remarkably his liverenzyms normalized. The ascites disappeared and he had no fever anymore. Also the alpha-fetoprotein deteriorated (Figure 1). The tumor was not progressive for a few years without any therapy.

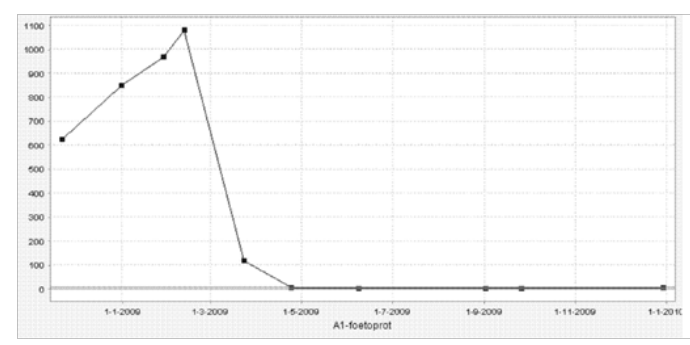

Figure I After surgery + RFA.

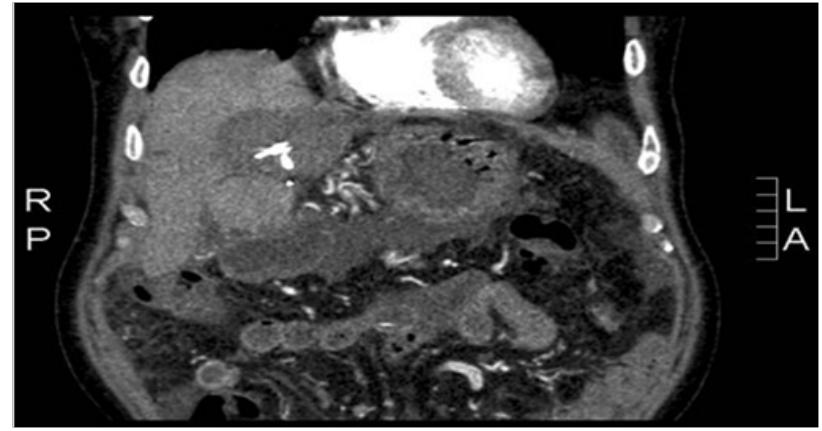

Figure 2 Portal vein thrombosis.

\section{Discussion}

In Egypt, schistosomiasis (mansoni) was the major cause of liver disease. From 1950s until the 1980s, the Egyptian Ministry of Health undertook large control campaigns using the standard treatment for schistosomiasis as a community-wide therapy.

When in the 1990s the diagnostic serology became available HCVinfection became the dominant cause of chronic liver disease in Egypt. Studies showed that co-infections of HCV with schistosomiasis caused more severe liver disease. ${ }^{1}$ Patients with $\mathrm{HCV}$ and schistosoma infection have a higher risk of cirrhosis and HCC. The influence of a strongyloides infection has not been clarified yet.

The combination of schistosomiasis and HCC has been identified as an adverse prognostic factor. But for the strongyloides infection this has not been found. There are a few case reports published about biliairy strongyloides infection, ${ }^{2-4}$ but only one with also portal vein thrombosis. ${ }^{5}$

In this case the tumor marker deteriorates after treatment of the strongyloides infection. The treatment seems to have a positive effect on the tumor. We suspect that the strongyloides larvae were situated in the bile ducts and lead to cholestasis and inflammation, which explains the fever and so leading to portal vein thrombosis. The portal vein thrombosis disappeared after treatment of the strongyloides infection and all of the patient's symptoms (Figure 3). 


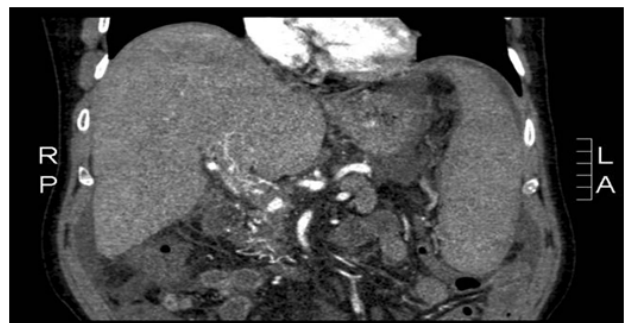

Figure $3 \mathrm{MRI}$ after strongyloides infection therapy.

\section{Conclusion}

This is a rare case of cholestasis and portal vein thrombosis associated with strongyloides infection and HCC. This case highlights the challenges of treatment of HCC with diagnosing underlying infections. In patient with suspected geographic exposure consider infections like strongyloidiasis.

\section{Acknowledgements}

None.

\section{Conflict of interest}

Author declares that there is no conflict of interest.

\section{References}

1. Strickland GT. Liver disease in Egypt: hepatitis C supersedes schistosomiasis as a result of iatrogenic and biological factors. Hepatology. 2006;43(5):915-922.

2. Hata H, Uba F, Okamoto Y, et al. A case of cholecystitis in which rhabdiform larvae of Strongyloides stercoralis were detected by duodenal intubation. J Kansai Med Univ. 1983;35(2):264-272.

3. Ishida $\mathrm{H}$, Inoue $\mathrm{T}$, Nishioka $\mathrm{Y}$, et al. A case of biliary strongyloidiasis. $J$ Jpn Soc Clin Surg. 1991;52(9):2151-2155.

4. Delarocque AE, Hadengue A, Degott C, et al. Biliary obstruction resulting from Strongyloides stercoralis infection. Report of a case. Gut. 1994;35(5):705-706.

5. Filkins LM, Gaston DC, Mathison B, et al. Biliary Strongyloides stercoralis with cholecystitis and extensive portal vein thrombosis. Open Forum Infect Dis. 2017;4(4):ofx217. 